\title{
消费端食物浪费: 影响与行动
}

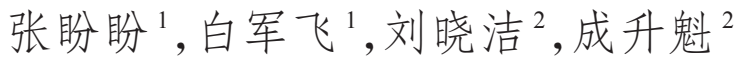 \\ (1. 中国农业大学经济管理学院, 北京 $100083 ; 2$. 中国科学院地理科学与资源研究所, 北京 100101)
}

\begin{abstract}
摘要：食物浪费已经成为全球性问题, 引发社会各界广泛关注。消费端是造成食物浪费的重 要环节, 且呈日趋严重的趋势。消费端的食物浪费主要分为家庭食物浪费和在外食物浪费; 消 费端的食物浪费对国家粮食安全、资源环境、社会经济发展等方面均产生不同的影响; 影响消 费端食物浪费的重要因素包括社会经济发展水平、文化因素、人口特征、消费者的行为与意识 等。目前对消费端食物浪费的研究多采用称重 (或估算) 和计量模型等方法, 仍需要通过发展 更合理的量化方法、建立更完整的理论模型、提出更完善的研究方法进行深人探究。此外, 减 少消费端食物浪费需要从政府、社会、个人等多角度出发, 促使多方利益相关者形成合力应对 消费端食物浪费问题。
\end{abstract}

关键词：食物浪费;消费端;影响与行动

食物浪费是全球性问题，引发了社会各界的广泛关注 ${ }^{[1]}$ 。据联合国粮农组织（FAO） 测算，全球每年约三分之一的食物（约为 13 亿 $\mathrm{t}$ ）在生产与消费过程中被浪费或损耗 ${ }^{[2]}$ 。 全球每年粮食浪费不仅造成了耕地和水资源的损耗，还导致了温室气体的排放 ${ }^{[3]}$ 。食物浪 费是一个复杂的、多学科的国际性问题，对全球可持续发展产生深远的影响 ${ }^{[4]}$ 。联合国粮 农组织（FAO）呼吁全球利益相关者团结起来合力应对食物浪费问题 ${ }^{[[]}$。

食物浪费与粮食安全密切相关 ${ }^{[4]}$, 并对社会、经济、环境等问题具有重大影响 ${ }^{[8]}$ 。未 来几十年, 无论是需求方面还是供给方面, 全球粮食系统可能将面临越来越大的压力 ${ }^{\left[{ }^{[}\right.}$。 必须尽量减少粮食系统相关的负面影响, 确保有足够的粮食以可持续发展的方式养活 （供养）世界日益增长的人口[7]。粮食的生产和销售需要消耗大量的能源、土地、水等资 源 ${ }^{[0-10]}$ 。食物浪费对粮食安全、资源环境、经济、社会等产生负面影响 ${ }^{[1]]}$, 被认为是亟需 解决的问题之一 ${ }^{[12]}$ 。

消费端是造成食物浪费的重要环节。消费端食物浪费比例占比较大 ${ }^{[13]}$ 。据估计大约 有 $35 \%$ 的食物损耗和浪费发生在消费端 ${ }^{[14]}$ 。在发达国家，消费者是食物浪费的最大来源 之一 ${ }^{[12]}$ 。在中国整个粮食供应链中, 消费端为粮食损失率占比最大的环节 ${ }^{[1]}$ 。食物浪费 发生在食物供应链的各个阶段，具有不同的特征和动机 ${ }^{[16]}$ 。目前，中国正处于工业化、 城镇化发展的关键时期, 食物浪费引发的资源环境压力已经成为制约社会经济健康持续 发展的重要瓶颈，减少食物浪费在一定程度上有利于保障中国社会经济健康持续发展。

\footnotetext{
收稿日期: 2018-06-13; 修订日期: 2018-10-11

基金项目：国家自然科学基金项目（71874178，71673272); 中国科学院先导项目（XDA19040303）; 重点研发计划 政府间国际科技创新合作重点专项（2016YFE0113100）；中国科学院重点部署项目（ZDBS-SSW-DQC）

作者简介: 张盼盼 (1989-), 女, 河南漯河人, 博士研究生, 研究方向为产业经济学和消费经济学。 E-mail: panpandazhang@163.com
}

通讯作者：刘晓洁（1972- ), 女, 山东潍坊人, 博士, 副研究员, 研究方向为资源可持续利用与区域发展。

E-mail: liuxj@igsnrr.ac.cn 


\section{1 食物浪费概念界定及现状}

\section{1 食物浪费概念界定}

食物浪费并没有统一的定义和概念界定 ${ }^{[17]}$ 。不同研究机构和学者对食物浪费的定义 有所差异。近年来，学术界对食物浪费的定义和估计方法尚未达成一致 ${ }^{[18]}$, 各类研究的 定义和方法不一，因此研究结果尚不具备直接的可比性 ${ }^{[19]}$ 。本文对学术界目前存在的食 物浪费定义 ${ }^{[4,18,20-25]}$ 进行归纳整理，具体见表 1 。

表 1 食物浪费的概念界定

Table 1 Definition of food waste

\begin{tabular}{|c|c|c|c|}
\hline 作者 & 发表年份 & 来源 & 定义 \\
\hline WRI ${ }^{[18]}$ & 2016 & 世界资源研究所（WRI） & $\begin{array}{l}\text { 食物损失和食物浪费 (FLW) 通常是指在食物供应链 } \\
\text { 各阶段中损失、减少的食物及/或相关不可食用部分 }\end{array}$ \\
\hline Thybery 等 ${ }^{[4]}$ & 2016 & $\begin{array}{l}\text { Resources, Conservation } \\
\text { and Recycling }\end{array}$ & $\begin{array}{l}\text { 食物浪费是指最初为人类消费而生产但后来被丢弃或 } \\
\text { 未被人类消费的食物, 包括变质的食物和丢弃时仍可 } \\
\text { 食用的部分 }\end{array}$ \\
\hline $\begin{array}{l}\text { European } \\
\text { Commission }^{[20]}\end{array}$ & 2016 & $\begin{array}{l}\text { 欧盟委员会（European } \\
\text { Commission） }\end{array}$ & $\begin{array}{l}\text { 食物浪费是指食物生产、分配和消费过程中产生的废 } \\
\text { 弃物 }\end{array}$ \\
\hline Buzby 等 ${ }^{[21]}$ & 2014 & 美国农业部（USDA） & $\begin{array}{l}\text { 食物浪费是食物损失的一个组成部分, 发生在可食用 } \\
\text { 食物未被消费时, 例如消费者餐盘中被丢弃的食物 }\end{array}$ \\
\hline Byker 等 ${ }^{[22]}$ & 2014 & $\begin{array}{l}\text { Journal of Nutrition } \\
\text { Education and Behavior }\end{array}$ & $\begin{array}{l}\text { 食物浪费是指在美国学校午餐计划中提供的可食用但 } \\
\text { 被浪费的食品和饮料 }\end{array}$ \\
\hline $\mathrm{FAO}^{[23]}$ & 2013 & FAO & 食物浪费是指用于人类消费的但被丢弃的那部分食物 \\
\hline Bond 等 ${ }^{[24]}$ & 2013 & Global Food Security & $\begin{array}{l}\text { 食物浪费通常指可食用的食物, 其目的是供人类消费 } \\
\text { 但是被丢弃、损耗等的部分, 不包括不可食用部分和 } \\
\text { 不合需求的部分 }\end{array}$ \\
\hline $\mathrm{FAO}^{[25]}$ & 1981 & FAO & $\begin{array}{l}\text { 食物浪费是指为人类消费而存在的, 但在食物供应链 } \\
\text { 的各个阶段被丢弃、降解或消耗的那部分食物 }\end{array}$ \\
\hline
\end{tabular}

本文认为，食物浪费（Food Waste）和食物损耗（Food Loss）是两个不同的概念。 食物浪费主要是指粮食收获后，由于对食物不合理使用而造成的可食用部分被丢弃。食 物损耗是指由于技术、管理手段等原因在收获、运输、储存等环节形成的一种不可避免 的食物损失。本文主要研究消费端的食物浪费，其中的食物是指可食用且非液体的部 分，不包括餐桌食物剩余量中的辅料、骨头等不可食用部分，也不包括食物剩余量中的 液体部分。根据场所不同，消费端食物浪费分为在家（家庭）食物浪费和在外食物浪费。

\section{2 食物浪费现状}

不同国家和地区食物浪费量有所差异。由美国农业部数据估算食物损失量和食物损 失价值，估计结果显示：在消费者层面，美国消费者 2008 年人均食物浪费量为 $124 \mathrm{~kg}$, 相当于人均损失 390 美元 ${ }^{[2]}$ 。据估计，加拿大每年浪费 270 亿美元的食物，其中有一半的 食物浪费发生在家庭层面 ${ }^{[27]}$ 。研究结果显示：从能量平衡角度来看，2005-2007年间瑞士 整个食物价值链中产生卡路里的 $48 \%$ 被浪费。若采取适当措施，这些损失中的一半是可 以避免的 ${ }^{[28]}$ 。芬兰整个国家 2010 年的食物浪费量为 1.2 亿 $\mathrm{kg}^{[29]}$ 。中国科学院地理科学与资 源研究所与世界自然基金会（WWF）联合发布的报告指出，仅 2015 年中国城市餐饮食 物浪费总量约为 1700 万 1800 万 $\mathrm{t}$ ，相当于全国粮食产量的 $3 \%{ }^{[30]}$ 。

消费端的家庭食物浪费研究主要集中在欧洲、美国等国家和地区。家庭食物浪费是 
欧盟食物浪费总量的重要贡献者 ${ }^{[31]}$ 。通过对芬兰家庭的调研，每年人均食物浪费量约为 $23 \mathrm{~kg}$, 每年每个家庭的食物浪费约为 $63 \mathrm{~kg}^{[29]}$ 。食物浪费占家庭食品支出的很大一部 分，研究结果表明 2008 年美国家庭食物损失价值相当于食物消费支出的 $10 \%$ ，超过平均 可支配收人的 $1 \%{ }^{[26]}$ 。据美国农业部（USDA）数据预测，美国人在外就餐食物支出占总 可支配收人的比例呈稳步上升趋势：1929年，美国人在外就餐食物支出占总可支配收人 的 $3.4 \%, 2014$ 年占比上升为 $5.4 \%$ 。人们的食物消费越来越多地发生在餐馆，家庭中的 食物浪费可能会转移到餐馆等商业部门 ${ }^{[4]}$ 。

消费者在外就餐比例不断增大 ${ }^{[33]}$ ，在外就餐食物浪费情况也愈发严重。随着经济的 发展和人民生活水平的提高，消费者在外就餐比例不断上升 ${ }^{[34]}$ 。据估计现阶段美国近一 半的食物支出用于在外就餐。1929年，美国居民在外食物消费支出为 2.7 亿美元； 2014 年在外食物消费支出上涨为 5580 亿美元，在外消费支出的年均增长率为 $6.47 \%$ 。在餐 饮业食物浪费调查中，消费者在不同类型餐馆中的食物浪费量不同 ${ }^{[35]}$ 。2014 年北京在校 学生平均食物浪费量为每餐 $130 \mathrm{~g}$, 占食物总量的 $21 \%{ }^{[36]}$ 。研究结果显示： 2010 年芬兰食 品服务行业中的食物约有 $20 \%$ 被浪费, 造成浪费的主要因素是自助服务和过度生产 ${ }^{[37]}$ 。

消费端食物浪费现状及成因尚不清晰。目前食物浪费数据存在高度的不一致 ${ }^{[38]}$ 。现 有研究中食物浪费的数据不足, 估算的差异性也很大 ${ }^{[13]}$ 。目前对消费端食物浪费研究的 数据有待进一步加强，由于一手数据的局限，使得消费端食物浪费的科学分析受到一定 影响。在对消费端食物浪费研究中, 需加强实地调研获取食物浪费一手数据, 确保开展 有针对性、科学性的研究工作。

\section{2 消费端食物浪费造成的影响}

\section{1 食物浪费对粮食安全的影响}

食物浪费事关粮食安全 ${ }^{[39]}$ 。全球约有 7.95 亿人遭受食物不足的困扰，其中有 7.8 亿居 住在发展中国家。要全方位确保粮食安全，需要采取更有针对性的政策措施，让各国相 关政策的制定者认识到在整个过程中面临挑战的多样性和复杂性 ${ }^{[40]}$ 。由于这种普遍存在 的粮食不安全现象, 减少食物浪费具有重要而深远的意义 ${ }^{[41]}$ 。为了实现食物供应链的可 持续发展，需要在制定可持续发展战略时充分考虑减少食物浪费相关问题 ${ }^{[42]}$ 。

在全球人口不断增长和资源日益匮乏的背景下，减少食物浪费将有助于改善未来的 粮食供应 ${ }^{[10,21,43]}$, 实现粮食系统的可持续性对全球发展至关重要 ${ }^{[4]}$ 。据联合国 2009 年估 计，世界人口到本世纪中叶将达到 91 亿，对粮食产量的需求将增长 $70 \%$ 。据联合国 2013 年报告显示，世界人口到 2050 年将达到 96 亿年]。为满足日益增长的粮食消费需求， 需要生产更多的粮食, 农业和其他有限的资源将面临更大的压力。有必要探索利用最少 量投人生产一定食物的方法，以便全球粮食系统在更小的环境成本下提供更好的营养 ${ }^{[4]}$ 。

减少食物浪费对粮食安全至关重要 ${ }^{[47]}$ 。相对于粮食市场有效供求决定的粮食产量, 粮食损失和浪费行为的发生则需要生产更多的粮食来满足市场需求。由于整个供应链的 粮食生产对环境产生影响，粮食损失和浪费加大了整个供应链中粮食生产对环境的影 响。因此, 保持当前粮食生产水平的同时减少食物浪费, 有助于满足全球范围内的粮食 需求。有研究表明, 如果将澳大利亚所有可避免的食物浪费捐献给慈善机构, 那么这些 曾经浪费掉的食物每年可养活 92.1 万人 ${ }^{[48]}$ 。可以说，一个地区减少食物浪费可能促进其 
他地区食物更好的利用 ${ }^{[49]}$ 。

\section{2 消费端食物浪费对资源环境的影响}

消费端食物浪费对资源环境具有负面影响 ${ }^{[2]}$ 。食物浪费造成资源的额外损耗 ${ }^{[15,50-51]}$, 并造成了一定的环境代价 ${ }^{[47,52-53]}$ 。运用 CHNS 数据研究表明, 中国家庭食物浪费对气候、 水和土地资源造成负面影响，2011年中国家庭人均每年浪费 $16 \mathrm{~kg}$ 食物，相当于 $40 \mathrm{~kg}$ 二 氧化碳的排放量、 $18 \mathrm{~m}^{3}$ 水的额外损耗 ${ }^{[54]}$ 。芬兰家庭 2010 年食物浪费产生的温室气体相当 于每年 10 万辆汽车二氧化碳的排放量 ${ }^{[5]}$ 。有学者利用生命周期法对美国食物浪费的环境 效应进行评估，结果显示：美国每年的食物浪费至少形成了 1.13 亿 $\mathrm{t}$ 的二氧化碳排放 ${ }^{[56]}$ 。 据估计，因食物损失和浪费而造成的资源损耗约为粮食生产耗费总淡水资源的 $24 \%$ 、总 耕地面积的 $23 \%$ 、全球化肥施用量的 $23 \%{ }^{[57]}$ 。在美国，生产被浪费的那部分食物需要耗 费美国总淡水消费量的 $25 \%$ 以上和 3 亿桶原油 ${ }^{[50]}$ 。全球范围内损失和浪费的食物相当于生 产这部分粮食所需资源的无谓损耗。

消费端食物浪费最小化有利于提高资源环境效益。消费端食物浪费最小化可以从数 量上减少食物浪费量，这将减少生产这部分粮食所需资源的无谓损耗，提高资源的使用 效益。预防食物浪费对环境影响很大 ${ }^{[49]}$, 例如减少食物浪费有利于温室气体的减排，有 效预防食物浪费可以减少包装废弃物, 并减少与食物生产、运输等相关的环境影响 ${ }^{[17]}$ 。全 球范围内人口的增长和消费模式的改变将导致全球食物需求的增长以及环境压力的增 大, 食物浪费对资源环境的影响需要引起重视。

\section{3 消费端食物浪费对社会经济的影响}

消费端食物浪费给食物消费者本身带来了经济损失 ${ }^{[47]}$ 。2010年芬兰家庭食物浪费的 经济价值相当于每人每年损失 70 欧元 ${ }^{[5]}$ 。食物浪费给社会也带来一定的损失。根据意大 利一家零售商店的食物浪费记录, 这家商店 2015 年产生的食物浪费量为 $70.6 \mathrm{t}$, 总价值 近 17 万欧元，浪费的食物主要为面包和新鲜的蔬菜、水果 ${ }^{[16]}$ 。相关研究表明，减少食物 浪费对不同国家产生的经济影响取决于一个国家的结构 ${ }^{[58]}$ 。了解食物浪费的经济成本可 能会促进消费者改善其消费行为，减少食物浪费而节省的资金被视为预防消费者食物浪 费行为的驱动因素 ${ }^{[59-60]}$ 。本文对食物浪费造成的经济损失 ${ }^{[9,16,21,23,26-27,29,55-56]}$ 进行归纳整理, 具 体见表 2 。

\section{3 消费端食物浪费产生的驱动因素}

食物浪费的产生是个人消费习惯、文化、政治、地理和经济等多重因素综合作用的 结果 ${ }^{[43]}$ 。食物浪费的驱动因素复杂, 因人而异、因年而异、因社会而异 ${ }^{[4]}$, 但对具体因素 的研究资料有限 ${ }^{[19]}$ 。有学者提出食物浪费属于 “奢侈” 消费的范畴，而“奢侈” 消费将 导致资源过度利用等问题 ${ }^{[1]}$ 。正视食物浪费研究的重要性, 探讨并科学分析消费端食物 浪费产生的驱动因素，可为制定食物可持续发展战略提供科学支撑。

\section{1 社会经济发展}

全球化促使饮食结构发生变化, 可能导致浪费更多的食物 ${ }^{[4]}$ 。城市化进程中需要更大 的食物供应系统与之相匹配 ${ }^{[13]}$, 随着收人水平的提高和城市化的快速推进, 消费端食物 浪费进一步加剧 ${ }^{[62]}$ 。

食物浪费行为存在较大的城乡差异性。城市居民的家庭食物浪费量远远高于农村居 
表 2 食物浪费的经济损失

Table 2 Economic loss of food waste

\begin{tabular}{|c|c|c|c|c|}
\hline 国家 & 发表年份 & 作者 & 期刊/机构 & 经济损失 \\
\hline 意大利 & 2017 & Cicatiello等 ${ }^{[16]}$ & $\begin{array}{l}\text { Resources, Conservation \& } \\
\text { Recycling }\end{array}$ & $\begin{array}{l}\text { 一家零售商店食物浪费 } 70.6 \mathrm{t} / \mathrm{a} \text {, 价值 } \\
\text { 近 } 17 \text { 万欧元/a }\end{array}$ \\
\hline 芬兰 & 2014 & Silvennoinen 等 ${ }^{[29]}$ & British Food Journal & $\begin{array}{l}23 \mathrm{~kg} /(\text { 人 · a }) \text {, 芬兰整个国家每年食 } \\
\text { 物浪费量为 } 1.2 \text { 亿 } \mathrm{kg}\end{array}$ \\
\hline 芬兰 & 2014 & Katajajuuri 等 ${ }^{[5]}$ & Journal of Cleaner Production & 70 欧元 $/($ 人 · a) \\
\hline 美国 & 2014 & Buzby 等 ${ }^{[2]]}$ & USDA Economic Research Service & 1616 亿美元/a，1249卡路里/(人 · a) \\
\hline 全球范围 & 2013 & $\mathrm{FAO}^{[23]}$ & FAO & 7500 亿美元/a \\
\hline 美国 & 2012 & Buzby 等 ${ }^{[26]}$ & Food Policy & 1656 亿美元/a，390美元/(人 · a) \\
\hline 美国 & 2011 & Venkat $^{[56]}$ & $\begin{array}{l}\text { International Journal on Food } \\
\text { System Dynamics }\end{array}$ & 1977 亿美元/a，643.3美元/(人 · a) \\
\hline 加拿大 & 2010 & Gooch 等 $^{[27]}$ & Value Chain Management Centre & 266亿美元/a \\
\hline 美国 & 2010 & Cuéllar等 ${ }^{[9]}$ & Environmental Science \& Technology & $2030 \pm 160$ 万亿 BTU 能源/年 \\
\hline
\end{tabular}

民的食物浪费量 ${ }^{[19]}$ 。随着收人的增加，食物支出占收人比例下降，食物浪费行为会相应 出现。在高收人国家，如美国，食物支出与其他费用（如住房）相比相对较低，食物浪 费现象相对普遍。研究表明, 在高收人国家, 家庭产生的食物浪费量占食物总浪费量的 一半，家庭食物浪费是造成食物浪费的最主要因素之一 ${ }^{[63]}$ 。

食物浪费随收人水平提高而增加。研究表明, 随着家庭收人的增长, 家庭饮食结构 发生变化并呈现多样化，具体表现为淀粉类主食占比下降，奶制品、肉类、鱼类、家禽 等比例增加 ${ }^{[13]}$; 家庭人均食物浪费量随人均国内生产总值的提高而增加 ${ }^{[38]}$ 。食物浪费在 一定程度上取决于食物价格、工资收人和非工资收人 ${ }^{[64]}$; 高收人家庭的食物浪费量高于 低收人家庭 ${ }^{[65]}$ 。

食品包装、饮食习惯、餐饮服务等多重因素可能间接造成食物浪费。随着社会经济 的发展和收人水平的提高, 人们对食物要求越来越精细化, 伴随着食品包装、饮食习 惯、餐饮服务的日趋多元化, 可能间接造成食物浪费。例如, 食品包装可以增加对产品 的保护, 便于温度控制并可防止在分配过程中发生的损坏 ${ }^{[6]}$ 。但已有研究指出, 家庭食 物浪费的 $20 \% \sim 25 \%$ 是由于食品包装因素造成的 ${ }^{[6]}$, 例如：包装太大、包装袋难以清空, 包装袋上面提供的 “最佳赏味期” 等 ${ }^{[6]}$ 。另外，消费者对食物新鲜度的关注也会造成食 物浪费 ${ }^{[8]}$; 食物供应模式、食堂服务质量、饮食习惯等因素也是间接影响食物浪费的重 要因素 ${ }^{[36]}$ 。

\section{2 文化因素}

文化因素既对饮食和营养方面起着至关重要的影响 ${ }^{[69-70]}$, 也对食物浪费的产生具有重 要的作用 ${ }^{[4]}$ 。可以说，食物浪费情况与一个社会的国情和文化规范有关 ${ }^{[7]}$ 。不同文化背景 的人们对食物的可食用部分的认识有所差异，并将被视为不可食用的部分予以丢弃 ${ }^{[4]}$ 。

消费者的食物浪费行为存在文化差异性。各国对食物文化和食物的态度不一样。例 如，法国人相对于美国人来说，更关注饮食的品质，更倾向于支持健康的饮食生活方 式 ${ }^{[69]}$ 。家庭购物习惯，特别是购物频率和购买商店的规模影响其食物浪费行为 ${ }^{[3]}$; 相对 于发达国家，发展中国家的消费者每次购买较少量的食物 ${ }^{[3]}$ ，这可能会减少食物浪费。

食物浪费受到文化相关因素的影响。对于食物零售商, 特别是超市, 由于对食物重 
量、形状、外观等质量具有严格的标准从而造成食物浪费 ${ }^{[2]}$ 。有研究表明，降低酒店餐盘 的物理尺寸将促使食物浪费减少 $19.5 \%{ }^{[72]}$ 。大尺寸将造成食物浪费和肥胖问题 ${ }^{[73]}$ 。促销活 动可能会造成更多的家庭食物浪费，因为消费者在促销情况下购买了比平时更多的食 物 ${ }^{[7]}$ 。处于旅游状态的消费者比非旅游状态的消费者浪费更多的食物 ${ }^{[3,75]}$ 。

\section{3 人口特征因素}

食物浪费行为与某些社会人口特征有关 ${ }^{[43]}$, 了解人口结构变化可以更好地了解其可 能带来的食物浪费变化 ${ }^{[4]}$ 。食物浪费行为受消费者人口特征因素的影响，例如年龄、收 人、性别、受教育水平等。目前关于哪些社会人口因素产生更多的浪费还没达成明确的 共识 ${ }^{[4]}$ 。

年龄是影响食物浪费的重要因素。不同年龄阶段的消费者食物浪费行为不同 ${ }^{[6]}$, 其 中年轻人比老年人浪费更多的食物 ${ }^{[6,76]}$ 。在澳大利亚, 食物浪费量随着年龄的增长而急剧 下降，18 24岁的受访者中有 38\%的受访者在两周内浪费了超过一定金额的新鲜食物， 而 70 岁以上的受访者中这个比例为 $7 \%$ 。研究表明, 65 岁以上人的食物浪费量远远低 于其他年龄阶段，一般这些年长的受访者具有不同生活经历，例如有些人经历过二战期 间食物短缺期，更加珍惜食物、明确反对食物浪费 ${ }^{[0]}$ 。

家庭构成和家庭规模显著影响家庭食物浪费 ${ }^{[65776-78]}$ 。例如，有孩子的家庭比没有孩子 的家庭浪费更多的食物, 成年人比小孩浪费更多的食物。家庭规模对人均食物浪费量有 显著影响, 研究发现单人的家庭人均食物浪费最大 ${ }^{[11]}$ 。同时, 也有研究认为, 家庭规模 对人均食物浪费量无显著差异，但有数据显示单个人的家庭产生的人均食物浪费量最大, 特别是独居女性产生的人均浪费量最大 ${ }^{[79]}$ 。家庭规模越大的人均食物浪费量越小 ${ }^{[67,77,80]}$ 。

收人水平显著影响消费者食物浪费行为。与高收人家庭相比, 低收人家庭的食物浪 费量较小 ${ }^{[76]}$, 食物浪费量随家庭收人水平的提高而增加 ${ }^{[80]}$ 。有研究表明, 收人越高的消 费者越容易产生食物浪费行为 ${ }^{[75]}$ 。然而, 另有研究发现收人水平和食物浪费之间没有显 著相关性 ${ }^{[78-79]}$ 。有研究认为，中低产家庭用于节省资金的食物消费策略实际上最终会导致 更多的食物浪费 ${ }^{[1]}$ 。

\section{4 消费者行为与意识因素}

联合国粮农组织（FAO）认为, 消费者的态度是影响家庭食物浪费的重要原因 ${ }^{[2]}$, 消 费者的个人偏好、价值观等会导致不同的食物浪费行为 ${ }^{[4]}$ 。消费者的感知行为控制是造成 食物浪费的主要驱动因素 ${ }^{[63]}$ 。例如, 食物浪费会使浪费者产生内疚感 ${ }^{[82]}$ 。消费者的行为 对减少食物浪费具有重要作用 ${ }^{[7,83]}$ 。食物浪费的相关意识行为已被证明可以减少食物浪 费，但在学术界并没有达成明确的共识 $[77]$ 。有研究表明，消费者的习惯和情绪对减少食 物浪费具有重要作用 ${ }^{[8]}$ 。消费者的态度、主观规范、感知行为控制等对家庭食物浪费具 有重要作用 ${ }^{[82]}$ 。

消费者食物的管理技能对食物浪费行为产生重要的影响 ${ }^{[12]}$ 。造成食物浪费的原因主 要有过度采购、过量购买、不恰当的食物保存方式等, 此外, 冲动购买、缺乏规划和偏 好大包装也是造成食物浪费的原因之一 ${ }^{[81]}$ 。消费者对食物需求预测的不精准也是造成食 物浪费的原因之一 ${ }^{[74]}$ 。家庭食物浪费主要由消费者在规划和购买食物方面的惯例驱动 的 ${ }^{[85]}$ 。食物浪费的产生与家庭购物习惯、食物准备行为、家庭垃圾管理技能有关，也与 食物相关的态度、理念和生活方式有关 ${ }^{[7]}$ 。人们缺乏对食物浪费相关知识的了解和关注 
程度 ${ }^{[3,86]}$, 购物阶段的不良规划导致过度供应、冲动购买和大量购买 ${ }^{[79]}$, 这在很大程度上 导致了食物浪费 ${ }^{[43]}$ 。

消费者对食物浪费相关知识的掌握与认识有利于减少食物浪费 ${ }^{[87-88]}$ 。有研究表明, 对 食物浪费问题有正确意识的消费者, 越容易减少食物浪费 ${ }^{[6]}$ 。有学者以招募的 540 名大学 生为研究对象，让其参与就餐计划并评估了信息干预是否会对其食物浪费行为产生影 响，结果显示简单的信息干预可有效减少 $15 \%$ 的食物浪费 ${ }^{[88}$ 。不同的信息对于减少食物 浪费产生不同的影响 ${ }^{[8-900}$, 例如, 消费者对食物浪费相关信息的了解有利于减少浪费, 但 当消费者得知浪费的食物可用于堆肥时食物浪费量不会显著减少 ${ }^{[80]}$; 向消费者提供食物 浪费相关的负面信息将会减少其食物浪费 ${ }^{[89]}$ 。

\section{4 消费端食物浪费量化及实证分析方法}

目前, 消费端食物浪费大多采用称重或估算的方式来量化。对食物浪费量进行量化 是制定行之有效的食物浪费管理政策的重要条件，也可用于评估食物浪费相关回收措施 和预防措施的实施效果 ${ }^{[4]}$ 。采用日记账的方式评估食物浪费情况是目前研究中采用的一种 方法。芬兰自然资源研究所的工作人员开展的项目研究芬兰食品服务部门的食物浪费数 量和组成 ${ }^{[3]}$, 通过对 51 个食品服务网点（包括学校、日托中心、餐厅等）可避免浪费的 食物的数量、类型和来源进行了调查 ${ }^{[3]}$ 。该研究采用日记账形式, 并在一周或一天时间 内对浪费的食物进行称重，结果表明食品服务行业的食物中约有 $20 \%$ 被浪费 ${ }^{[3]}$ 。然而， 食物浪费的定义不清, 并且缺乏合理的量化方法, 导致了食物浪费数据的低效性。

有部分研究运用计量模型对食物浪费进行实证分析。有学者运用Probit模型分析消 费者在购物前和消费阶段食物浪费的影响因素 ${ }^{[8]}$ 。近年来，有学者运用干预试验的方法 来研究食物浪费问题 ${ }^{[00-91]}$, 例如, 以德国家庭为研究对象, 运用方差分析, 结果表明干预 策略对食物浪费行为有影响 ${ }^{[2]}$ 。有学者运用元分析和系统评估的方法, 估算美国食物浪 费总量 ${ }^{[8]}$ 。针对食物损失和浪费的现有研究方法存在较多可改进之处 ${ }^{[93]}$ 。本文对食物浪费 定量化评估和实证分析方法进行归纳梳理，具体见表 3 。

目前学术界关于食物浪费研究的理论模型欠缺, 研究方法具有一定的局限性, 尚未 形成完整的理论体系和研究方法 ${ }^{[94]}$ 。对食物浪费的研究大多采用综述性分析或描述性统 计分析，学术界需加大对食物浪费科学研究方法的探索和研发。

\section{5 减少消费端食物浪费的行动}

食物浪费研究文献中, 针对食物浪费的政策建议主要从国家、社会、个人三方面提 出。政府、社会利益相关者和零售商应采取行动减少食物浪费, 并且各方之间的协作是 很重要的 ${ }^{[1]}$ 。为了减少食物浪费, 需要确定在粮食供应链上影响食物浪费的主要原因。 在粮食供应链上的所有参与者应该共同努力分担责任减少食物浪费 ${ }^{[9]}$ 。有研究以学校为 研究对象, 对食堂管理人员、家长、教师等人员进行访谈, 结果显示食物浪费的利益相 关者均认为应该采取措施减少食物浪费。减少食物浪费的措施包括积极鼓励孩子消费、 提供较小份量的食物、捐赠未食用的食物等 ${ }^{[97]}$ 。全球消费者、企业、政府和机构共同承 担减少食物浪费的责任, 珍惜食物、减少浪费并优化资源 ${ }^{[89]}$ 。 
表 3 食物浪费量化和实证分析方法

Table 3 Food waste quantification and empirical analysis

\begin{tabular}{|c|c|c|c|c|}
\hline 区域 & 发表年份 & 作者 & 估计方法 & 期刊/机构 \\
\hline 中国 & 2018 & 张盼盼等 ${ }^{[90]}$ & Logit模型、Tobit模型 & 自然资源学报 \\
\hline 中国 & 2018 & 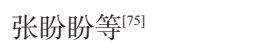 & Tobit模型 & 资源科学 \\
\hline 欧洲 & 2017 & Campoy-Muñoz等 ${ }^{[58]}$ & CGE模型 & Resources, Conservation \& Recycling \\
\hline 英国 & 2017 & Russell等 ${ }^{[84]}$ & 分层多元回归 & Resources, Conservation \& Recycling \\
\hline \multirow[t]{2}{*}{ 英国 } & 2017 & Young 等 ${ }^{[1]}$ & 方差分析（ANOVA） & Resources, Conservation \& Recycling \\
\hline & 2017 & Qi 等[89] & $\begin{array}{l}\text { 方差分析 (ANOVA)、Tobit } \\
\text { 模型、OLS-IV、LPM-IV }\end{array}$ & The 2017 ASSA Annual Meeting \\
\hline 意大利 & 2017 & Cicatiello 等 ${ }^{[16]}$ & Meta分析 & Resources, Conservation \& Recycling \\
\hline 丹麦 & 2016 & Stancu等 ${ }^{[63]}$ & 结构方程模型 & Appetite \\
\hline 德国 & 2016 & Schmidt $^{[92]}$ & 方差分析（ANOVA） & Resources, Conservation \& Recycling \\
\hline 全球 & 2016 & Chalak等[95] & OLS 回归 & Waste Management \\
\hline 美国 & 2016 & Ellison 等 ${ }^{[64]}$ & OLS 回归、Logit模型 & The 2016 AAEA Conference Paper \\
\hline 意大利 & 2015 & Principato 等 ${ }^{[68]}$ & Probit模型 & British Food Journal \\
\hline 美国 & 2015 & Thyberg 等 ${ }^{[8]}$ & Meta分析 & Environment Science \& Technology \\
\hline 中国 & 2013 & 胡越等 ${ }^{[52]}$ & GTAP模型 & 中国人口・资源与环境 \\
\hline
\end{tabular}

目前有学者提出从国家的角度来预防食物浪费。明确的立法、政策对减少食物浪费 是有效用的 ${ }^{[95]}$ 。美国农业部（USDA）推行了若干减少食物浪费的措施，例如通过对捐赠 者进行税收优惠和责任保护等 ${ }^{[4]}$ 。目前在美国，没有广泛或明显的政治或社会动力来防止 食物浪费 ${ }^{[2]}$ 。中国政府推行了若干减少食物浪费的举措, 其中包括 2014 年国务院办公厅 印发的《中国食物与营养发展纲要（2014-2020年）》中提出 “厉行节约、反对浪费”; 2014年中共中央办公厅、国务院办公厅印发的《关于厉行节约反对食品浪费的意见》中 指出 “杜绝公务活动用餐浪费” “减少各环节粮食损失浪费”; 2016年国家发改委、中宣 部、科技部等部门联合发布的《关于促进绿色消费的指导意见》中明确提出 “开展反食 品浪费行动”。研究表明, 只有通过政府监管产生食物浪费的机构, 即食物生产企业和家 庭，食物浪费才能大幅度减少 ${ }^{[1]}$ 。在居民社区中提高服务质量和基础设施建设有助于促进 居民有效参与废物管理活动，从而改善食物浪费行为 ${ }^{[99]}$ 。

应从社会组织机构角度改善食物浪费行为。近年来，政府、个人、民间社会组织制 定了减少食物浪费的方案, 其中民间社会组织成为治理食物浪费的重要机构 ${ }^{[1]}$ 。然而, 民间社会组织对治理食物浪费和保障粮食安全的效力尚不明确 ${ }^{[1]}$ 。以洛杉矶食物救援为 例，民间社会组织虽然拯救了一些食物，但并没有找到造成食物浪费和粮食不安全的根 本原因 ${ }^{[11]}$ 。有研究针对瑞典 3 种类型食物（面包、水果和蔬菜、牛奶）的相关回收协议 (TBA）进行分析，结果表明 TBA 对食物浪费具有重要影响 ${ }^{[100]}$ 。在 $\mathrm{NGO}$ 组织的食物救援 活动中, 有研究表明澳大利亚组织每投人 1 美元则有价值 5.71 美元的可食用食物免于被 浪费 ${ }^{[48]}$ 。

减少食物浪费需从消费者个人人手。食物浪费归根到底是消费者行为选择的结果， 任何减少食物浪费的策略都必须落实到个体消费者 ${ }^{[75]}$ 。需要深人了解人们浪费食物的动 机以及有关减少食物浪费的相关知识 ${ }^{[3]}$ 。零售商和餐馆应采取措施减少食物浪费 ${ }^{[87]}$ 。 
强调食物浪费的经济影响，节约金钱被发现是减少食物浪费的强大动力 ${ }^{[59-60]}$ 。有研究表 明，提高人们对食物浪费数量的认识程度有利于减少其食物浪费行为 ${ }^{[101]}$ 。为人们提供食 物相关的教育培训和技能训练, 能够帮助人们更好地认识食物安全, 这对预防食物浪费 是必不可少的。

目前学术界关于减少消费端食物浪费的实施方案较少，相关的实施方案评定缺乏， 未来消费端食物浪费减量的政策措施和行动模式有待加强。在现有研究的基础上，借鉴 消费端已有的政策措施和管理模式，本文认为应从以下几方面提出消费端食物浪费减量 化的对策建议：第一，在国家层面明确立法，构建有效政策；第二，充分发挥社会组织 机构的作用, 加大宣传力度; 第三, 通过对消费者进行培训、提高意识等方式, 倡导绿 色消费理念和模式。

\section{6 讨论}

基于消费端食物浪费的影响与行动研究，本文针对食物浪费进行如下讨论：

（1）应加快完善食物浪费研究的理论及实证分析方法，加大对食物浪费科学研究方 法的探索和研发。逐步统一食物浪费概念及内涵的界定，规范食物浪费理论分析、实证 分析的模型及方法; 逐步制定食物浪费一手数据收集的工作流程与标准，有利于准确认 识到全球各地食物浪费的现状及差异。

（2）系统分析食物浪费的资源环境代价，深人研究食物浪费及其资源环境效用问 题，探索资源节约、环境友好型的可持续发展模式，具有重大的科学价值和社会意义。 与此同时，如何处理好食物浪费问题与建立资源节约、环境友好型社会目标之间的关系 存在重大挑战。相关研究应根据中国食物浪费面临的资源环境问题, 提出具有科学价值 的、适用于中国国情的食物浪费减量化、资源化目标和对策建议。

（3）食物浪费问题为多学科相互融合研究创造平台。食物浪费研究涉及多个学科研 究领域, 在融合经济学、社会学、心理学、生态学等相关学科及知识的基础上, 研究者 需认真汲取各领域专家学者的意见和建议，借鉴已有的研究成果，积极从各领域、各角 度综合分析食物浪费相关问题。

（4）食物浪费的深人研究有利于引发新时代保障粮食安全新路径的思考。从全球角 度来看：人口数量的增加引发对食物安全的担忧及考验。粮食安全是一国立足世界的基 本保障。目前，中国正处于经济快速发展、城镇化水平不断提高的关键时期，粮食的绝 对安全是对中国经济发展的有力保障，消费端的食物浪费在一定程度上制约中国经济社 会的良好健康发展。食物浪费的相关研究成果，可为减少食物浪费提供科学对策，从而 为保障粮食安全开辟新的潜力。古人云：一籿一饭当思来之不易，半丝半缕恒念物力维 艰。减少食物浪费既是中华民族的传统美德，又有利于提高全社会的福祉。

\section{参考文献(References):}

[1] 成升鬼, 白军飞, 金钟浩, 等. 笔谈: 食物浪费. 自然资源学报, 2017, 32(4): 529-538. [CHENG S K, BAI J F, JIN Z H, et al. Reducing food loss and food waste: Some personal reflections. Journal of Natural Resources, 2017, 32(4): 529538.]

[2] GUSTAVSSON J, CEDERBERG C, SONESSON U, et al. Global food losses and food waste: Extent, causes and preven- 
tion. FAO: Rome, Italy, 2011.

[3] FAO. Food wastage footprint-full cost accounting. FAO: Rome, Italy, 2014.

[4] THYBERG K L, TONJES D J. Drivers of food waste and their implications for sustainable policy development. Resources, Conservation and Recycling, 2016, 106: 110-123.

[5] FAO. FAO and messe düsseldorf lead the save food: Global initiative on food waste reduction. http://www.fao.org/savefood/background/en/. FAO: Rome, Italy, 2011.

[6] GODFRAY H C J, GARNETT T. Food security and sustainable intensification. Philosophical Transactions of the Royal Society B, 2014, 369: 20120273.

[7] TILMAN D, FARGIONE J, WOLFF B, et al. Forecasting agriculturally driven global environmental change. Science, 2001, 292(5515): 281-284.

[8] THYBERG K L, TONJES D J, GUREVITCH J. Quantification of food waste disposal in the United States: A meta-analysis. Environment Science \& Technology, 2015, 49: 13946-13953.

[9] CUÉLLAR A D, WEBBER M E. Wasted food, wasted energy: The embedded energy in food waste in the United States. Environmental Science \& Technology, 2010, 44(16): 6464-6469.

[10] GODFRAY H C J, BEDDINGTON J R, CRUTE I R, et al. Food security: The challenge of feeding 9 billion people. Science, 2010, 327(5967): 812-818.

[11] WARSHAWSKY D N. The devolution of urban food waste governance: Case study of food rescue in Los Angeles. Cities, 2015, 49: 26-34.

[12] ASCHEMANN-WITZEL J, HOOGE I, AMANI P, et al. Consumer-related food waste: Causes and potential for action. Sustainability, 2015, 7: 6457-6477.

[13] PARFITT J, BARTHEL M, MACNAUGHTON S. Food waste within food supply chains: Quantification and potential for change to 2050. Philosophical Transactions of the Royal Society B, 2010, 365: 3065-3081.

[14] LIPINSKI B, HANSON C, LOMAX J, et al. Installment 2 of "creating a sustainable food future": Reducing food loss and waste. http: //www. worldresourcesreport. org. World Resources Institute, Washington, DC, 2013.

[15] LIU J, LUNDQVIST J, WEINBERG J, et al. Food losses and waste in china and their implication for water and land. Environmental Science \& Technology, 2013, 47: 10137-10144.

[16] CICATIELlO C, FRANCO S, PANCINO B, et al. The dark side of retail food waste: Evidences from in-store data. Resources, Conservation \& Recycling, 2017, 125: 273-281.

[17] SCHOTT A B S, CÁNOVAS A. Current practice, challenges and potential methodological improvements in environmental evaluations of food waste prevention: A discussion paper. Resources, Conservation and Recycling, 2015, 101: 132-142.

[18] WRI. Food loss and waste accounting and reporting standard. http://www.wri.org/publication/flwstandard. World Resources Institute, 2016.

[19] LEBERSORGER S, SCHNEIDER F. Discussion on the methodology for determining food waste in household waste composition studies. Waste Management, 2011, 31: 1924-1933.

[20] European Commission. Reducing food waste: The EU's response to a global challenge. http://europa.eu/rapid/press-release_MEMO-16-3989_en.htm. European Commission, 2016.

[21] BUZBY J C, WELLS H F, HYMAN J. The estimated amount, value and calories of postharvest food losses at the retail and consumer levels in the United States. Washington, DC, USA: USDA Economic Research Service, 2014.

[22] BYKER C J, FARRIS A R, MARCENELLE M, et al. Food waste in a school nutrition program after implementation of new lunch program guidelines. Journal of Nutrition Education and Behavior, 2014, 46(5): 406-411.

[23] FAO. Food wastage footprint impacts on natural resources. Rome, Italy: FAO, 2013.

[24] BOND M, MEACHAM T, BHUNNOO R, et al. Food waste within global food systems. https://www.foodsecurity.ac uk/. Global Food Security, 2013.

[25] FAO. Food loss prevention in perishable crops. Rome, Italy: FAO, 1981.

[26] BUZBY J C, HYMAN J. Total and per capita value of food loss in the United States. Food Policy, 2012, 37: 561-570.

[27] GOOCH M, FELFEL A, MARENICK N. Food waste in Canada. Value Chain Management Centre, Canada, 2010. 
[28] BERETTA C, STOESSEL F, BAIER U, et al. Quantifying food losses and the potential for reduction in Switzerland. Waste Management, 2013, 33: 764-773.

[29] SILVENNOINEN K, KATAJAJUURI J-M, HARTIKAINEN H, et al. Food waste volume and composition in Finnish households. British Food Journal, 2014, 116(6): 1058-1068.

[30] 成升鬼, 金钟浩, 刘刚, 等. 中国城市餐饮食物浪费报告. 北京: 世界自然基金会, 中国科学院地理科学与资源研究 所, 2018. [CHENG S K, JIN Z H, LIU G, et al. Report on catering industry food waste in Chinese cities. Beijing: WWF, Institute of Geographic Sciences and Natural Resources Research, CAS, 2018.]

[31] JÖRISSEN J, PRIEFER C, BRÄUTIGAM K-B, et al. Food waste generation at household level: Results of a survey among employees of two European research centers in Italy and Germany. Sustainability, 2015, 7: 2695-2715.

[32] USDA. ERS food expenditures: Table 1 food and alcoholic beverages: Total expenditures and Table 8 food expenditures by families and individuals as a share of disposable personal money income. https://www.ers.usda.gov/data-products/ food-expenditures/food-expenditures/. USDA Economic Research Service, 2013.

[33] STEWART H, YEN S T. Changing household characteristics and the away-from-home food market: A censored equation system approach. Food Policy, 2004, 29: 643-658.

[34] LIU H, WAHL T I, SEALE JR J L, et al. Household composition, income, and food-away-from-home expenditure in urban China. Food Policy, 2015, 51: 97-103.

[35] WANG L E, LIU G, LIU X, et al. The weight of unfinished plate: A survey based characterization of restaurant food waste in Chinese cities. Waste Management, 2017, 66: 3-12.

[36] LIU Y, CHENG S K, LIU X J, et al. Plate waste in school lunch programs in Beijing, China. Sustainability, 2016, 8: 1288.

[37] SILVENNOINEN K, HEIKKILÄ L, KATAJAJUURI J-M, et al. Food waste volume and origin: Case studies in the Finnish food service sector. Waste Management, 2015, 46: 140-145.

[38] XUE L, LIU G, PARFITT J, et al. Missing food, missing data? A critical review of global food losses and food waste data. Environment Science \& Technology, 2017, 51: 6618-6633.

[39] STENMARCK Å, JENSEN C, QUESTED T, et al. Estimates of European food waste levels. Stockholm: European Commission, 2016.

[40] FAO, IFAD, WFP. The state of food insecurity in the world 2015. Rome, Italy: FAO, IFAD, WFP, 2015.

[41] GJERRIS M, GAIANI S. Household food waste in Nordic countries: Estimations and ethical implications. Nordic Journal of Applied Ethics, 2013, 7(1): 6-23.

[42] MARTINDALE W. Using consumer surveys to determine food sustainability. British Food Journal, 2014, 116(7): 11941204.

[43] PEARSON D, MINEHAN M, WAKEFIELD-RANN R. Food waste in Australian households: Why does it occur. The Australasian-Pacific Journal of Regional Food Studies, 2013, 3: 118-132.

[44] GARNETT T. Three perspectives on sustainable food security: Efficiency, demand restraint, food system transformation. What role for life cycle assessment?. Journal of Cleaner Production, 2014, 73: 10-18.

[45] FAO. Feeding the world, eradicating hunger. http://www. fao. org/fileadmin/templates/wsfs/Summit/WSFS_Issues_papers/WSFS_Background_paper_Feeding_the_world.pdf. World Summit on Food Security, Rome, Italy, 2009.

[46] United Nations. World population prospects, the 2012 revision. http://www.un.org/en/development/desa/publications/ world- population- prospects- the-2012- revision.html. New York, USA: Department of Economic and Social Affairs, 2013.

[47] BRANCOLI P, ROUSTA K, BOLTON K. Life cycle assessment of supermarket food waste. Resources, Conservation and Recycling, 2017, 118: 39-46.

[48] REYNOLDS C J, PIANTADOSI J, BOLAND J. Rescuing food from the organics waste stream to feed the food insecure: An economics and environmental assessment of Australian food rescue operations using environmentally extended waste input-output analysis. Sustainability, 2015, 7: 4707-4726.

[49] GENTIL E C, GALLO D, CHRISTENSEN T H. Environmental evaluation of municipal waste prevention. Waste Management, 2011, 31: 2371-2379. 
[50] HALL K D, GUO J, DORE M, et al. The progressive increase of food waste in America and its environmental impact. Plos One, 2009, 4(11): e7940, 1-6.

[51] 张丹, 成升鬼, 高利伟, 等. 城市餐饮业食物浪费的生态足迹: 以北京市为例. 资源科学, 2016, 38(1): 10-18. [ZHANG D, CHENG S K, GAO L W, et al. Ecological footprint of catering industry food waste in Beijing. Resources Science, 2016, 38(1): 10-18.]

[52] 胡越, 周应恒, 韩一军, 等. 减少食物浪费的资源及经济效应分析. 中国人口・资源与环境, 2013, 23(12): 150-155. [HU Y, ZHOU Y H, HAN Y J, et al. Resources and economic effects analysis of reducing food waste. China Population, Resources and Environment, 2013, 23(12): 150-155.]

[53] 成升鬼, 高利伟, 徐增让, 等. 对中国餐饮食物浪费及其资源环境效应的思考. 中国软科学, 2012, (7): 106-114. [CHENG S K, GAO L W, XU Z R, et al. Food waste in catering industry and its impacts on resources and environment in China. China Soft Science, 2012, (7): 106-114.]

[54] SONG G, LI M, SEMAKULA H M, et al. Food consumption and waste and the embedded carbon, water and ecological footprints of households in China. Science of the Total Environment, 2015, 529: 191-197.

[55] KATAJAJUURI J-M, SILVENNOINEN K, HARTIKAINEN H, et al. Food waste in the Finnish food chain. Journal of Cleaner Production, 2014, 73: 322-329.

[56] VENKAT K. The climate change and economic impacts of food waste in the United States. International Journal on Food System Dynamics, 2011, 2(4): 431-446.

[57] KUMMU M, DE MOEL H, PORKKA M, et al. Lost food, wasted resources: Global food supply chain losses and their impacts on freshwater, cropland, and fertilizer use. Science of the Total Environment, 2012, 438: 477-489.

[58] CAMPOY-MUÑOZ P, CARDENETE M A, DELGADO M C. Economic impact assessment of food waste reduction on European countries through social accounting matrices. Resources, Conservation and Recycling, 2017, 122: 202-209.

[59] GRAHAM-ROW E, JESSOP D C, SPARKS P. Identifying motivations and barriers to minimizing household food waste. Resources, Conservation and Recycling, 2014, 84: 15-23.

[60] QUESTED T E, MARSH E, STUNEL D, et al. Spaghetti soup: The complex world of food waste behaviours. Resources, Conservation and Recycling, 2013, 79: 43-51.

[61] BLAIR D, SOBAL J. Luxus consumption: Wasting food resources through overeating. Agriculture and Human Values, 2006, 23: 63-74.

[62] LIU G. Food losses and food waste in China: A first estimate. http://dx.doi.org/10.1787/5jz5sq51731q-en. OECD Food, Agriculture and Fisheries Papers, 2014.

[63] STANCU V, HAUGAARD P, LÄHTEENMÄKI L. Determinants of consumer food waste behavior: Two routes to food waste. Appetite, 2016, 96: 7-17.

[64] ELLISON B, LUSK J L. Examining Household Food Waste Decisions: A vignette approach. Jayson Lusk, 2016.

[65] HAMILTON C, DENNISS R, BAKER D. Wasteful consumption in Australia. Canberra, Australia: The Australia Institute, 2005.

[66] VERGHESE K, LEWIS H, LOCKREY S, et al. Packaging's role in minimizing food loss and waste across the supply Chain. Packaging Technology and Science, 2015, 28: 603-620.

[67] WILLIAMS H, WIKSTRÖM F, OTTERBRING T, et al. Reasons for household food waste with special attention to packaging. Journal of Cleaner Production, 2012, 24: 141-148.

[68] PRINCIPATO L, SECONDI L, PRATESI C A. Reducing food waste: An investigation on the behaviour of Italian youths. British Food Journal, 2015, 117(2): 731-748.

[69] ROZIN P. The meaning of food in our lives: A cross-cultural perspective on eating and well-being. Journal of Nutrition Education and Behavior, 2005, 37(2): S107-S112.

[70] SOBAL J. Cultural comparison research designs in food, eating, and nutrition. Food Quality and Preference, 1998, 9(6): 385-392.

[71] WILSON D C. Stick or carrot? The use of policy measures to move waste management up the hierarchy. Waste Management \& Research, 1996, 14: 385-398.

[72] KALLBEKKEN S, SÆLEN H. 'Nudging' hotel guests to reduce food waste as a win-win environmental measure. Eco- 
nomics Letters, 2013, 119: 325-327.

[73] YOUNG L R, NESTLE M. The contribution of expanding portion sizes to the US obesity epidemic. American Journal of Public Health, 2002, 92(2): 246-249.

[74] MENA C, ADENSO-DIAZ B, YURT O. The causes of food waste in the supplier-retailer interface: Evidences from the UK and Spain. Resources, Conservation and Recycling, 2011, 55: 648-658.

[75] 张盼盼, 王灵恩, 白军飞, 等. 旅游城市餐饮消费者食物浪费行为研究. 资源科学, 2018, 40(6): 1186-1195. [ZHANG P P, WANG L E, BAI J F, et al. The food waste behavior of catering consumers from a tourism perspective. Resources Science, 2018, 40(6): 1186-1195.]

[76] COX J, DOWNING P. Food behaviour consumer research: Quantitative phase. Banbury, UK: WRAP, 2007.

[77] PARIZEAU K, VON-MASSOW M, MARTIN R. Household-level dynamics of food waste production and related beliefs, attitudes, and behaviours in Guelph, Ontario. Waste Management, 2015, 35: 207-217.

[78] WENLOCK R W, BUSS D H, DERRY B J, et al. Household food wastage in Britain. British Journal of Nutrition, 1980, 43: $53-70$.

[79] KOIVUPURO H K, HARTIKAINEN H, SILVENNOINEN K, et al. Influence of socio-demographical, behavioural and attitudinal factors on the amount of avoidable food waste generated in Finnish households. International Journal of Consumer Studies, 2012, 36: 183-191.

[80] BAKER D, FEAR J, DENNISS R. What a waste: An analysis of household expenditure on food. Canberra, Australia: The Australia Institute, 2009.

[81] PORPINO G, PARENTE J, WANSINK B. Food waste paradox: Antecedents of food disposal in low income households. International Journal of Consumer Studies, 2015, 39: 619-629.

[82] GRAHAM-ROW E, JESSOP D C, SPARKS P. Predicting household food waste reduction using an extended theory of planned behaviour. Resources, Conservation and Recycling, 2015, 101: 194-202.

[83] FARR-WHARTON G, FOTH M, CHOI J H. Identifying factors that promote consumer behaviours causing expired domestic food waste. Journal of Consumer Behaviour, 2014, 13: 393-402.

[84] RUSSELL S V, YOUNG C W, UNSWORTH K L, et al. Bring habits and emotions into food waste behaviour. Resources, Conservation \& Recycling, 2017, 125: 107-114.

[85] STEFAN V, HERPEN E V, TUDORAN A A, et al. Avoiding food waste by Romanian consumers: The importance of planning and shopping routines. Food Quality and Preference, 2013, 28: 375-381.

[86] BUZBY J C, HYMAN J, STEWART H, et al. The value of retail-and consumer-level fruit and vegetable losses in the United States. The Journal of Consumer Affairs, 2011, 45(3): 492-515.

[87] NEFF R A, SPIKER M L, TRUANT P L. Wasted food: US consumers' reported awareness, attitudes, and behaviors. Plos One, 2015, 10: e0127881.

[88] WHITEHAIR K J, SHANKLIN C W, BRANNON L A. Written messages improve edible food waste behaviors in a university dining facility. Journal of the Academy of Nutrition and Dietetics, 2013, 113(1): 63-69.

[89] QI D, ROE B E. Foodservice composting crowds out consumer food waste reduction behavior in a dining experiment. The 2017 ASSA Annual Meeting, Chicago, 2017.

[90] 张盼盼, 白军飞, 成升鬼, 等. 信息干预是否影响食物浪费?: 基于餐饮业随机干预试验. 自然资源学报, 2018, 33(8): 1439-1450. [ZHANG P P, BAI J F, CHENG S K, et al. Does information intervention affect food waste?: Randomized controlled trials in catering industry. Journal of Natural Resources, 2018, 33(8): 1439-1450.]

[91] YOUNG W, RUSSELL S V, ROBINSON C A, et al. Can social media be a tool for reducing consumers' food waste? A behaviour change experiment by a UK retailer. Resources, Conservation and Recycling, 2017, 117: 195-203.

[92] SCHMIDT K. Explaining and promoting household food waste-prevention by an environment psychological based intervention study. Resources, Conservation and Recycling, 2016, 111: 53- 66.

[93] CHABOUD G. Assessing food losses and waste with a methodological framework: Insights from a case study. Resources, Conservation and Recycling, 2017, 125: 188-197.

[94] 王灵恩, 成升鬼, 刘刚, 等. 中国食物浪费研究的理论与方法探析. 自然资源学报, 2015, 30(5): 715-724. [WANG L E, CHENG S K, LIU G, et al. Study on theories and methods of Chinese food waste. Journal of Natural Resources, 
2015, 30(5): 715-724.]

[95] CHALAK A, ABOU-DAHER C, CHAABAN J, et al. The global economic and regulatory determinants of household food waste generation: A cross-country analysis. Waste Management, 2016, 48: 418-422.

[96] GÖBEL C, LANGEN N, BLUMENTHAL A, et al. Cutting food waste through cooperation along the food supply chain. Sustainability, 2015, 7: 1429-1445.

[97] BLONDIN S A, DJANG H C, METAYER N, et al. "It's just so much waste." A qualitative investigation of food waste in a universal free school breakfast program. Public Health Nutrition, 2014, 18(9): 1565-1577.

[98] FINN S M. Valuing our food: Minimizing waste and optimizing resources. Zygon, 2014, 49(4): 992-1008.

[99] RISPO A, WILLIAMS I D, SHAW P J. Source segregation and food waste prevention activities in high-density households in a deprived urban area. Waste Management, 2015, 44: 15-27.

[100] ERIKSSON M, GHOSH R, MATTSSON L, et al. Take-back agreements in the perspective of food waste generation at the supplier-retailer interface. Resources, Conservation and Recycling, 2017, 122: 83-93.

[101] MILIUTE-PLEPIENE J, PLEPYS A. Does food sorting prevents and improves sorting of household waste? A case in Sweden. Journal of Cleaner Production, 2015, 101: 182-192.

\title{
Food waste at the consumer segment: Impact and action
}

\author{
ZHANG Pan-pan ${ }^{1}$, BAI Jun-fei ${ }^{1}$, LIU Xiao-jie ${ }^{2}$, CHENG Sheng-kui ${ }^{2}$ \\ (1. College of Economics and Management, China Agricultural University, Beijing 100083, China; \\ 2. Institute of Geographic Sciences and Natural Resources Research, CAS, Beijing 100101, China)
}

\begin{abstract}
Food waste has become a global problem, which causes widespread concern from the public. The consumption segment is an important part of food waste, and it is increasingly serious. Food waste at the consumer segment is mainly divided into household food waste and food waste outside home. Food waste at the consumer segment has different impacts on national food security, resources and environment, and social and economic development. Food waste at the consumption segment is affected by factors such as economic and social development, cultural background, demographic characteristics, and consumer behavior and awareness. At present, the study of food waste at the consumer segment mostly uses methods such as weighing (or estimating) and measurement model. It still needs to be further explored by developing reasonable quantitative methods, establishing complete theoretical models and proposing concrete research methods. In addition, to reduce food waste at the consumer segment, we should encourage multi-stakeholders to make a joint effort to deal with food waste at the consumer segment from the perspectives of government, society, individuals and other angles.
\end{abstract}

Keywords: food waste; consumer segment; impact and action 